\title{
COCCIDIOSIS INTESTINAL EN NIÑOS ADMITIDOS EN UN HOSPITAL DE PERÚ Y COMPARACIÓN DE DOS MÉTODOS PARA LA DETECCIÓN DEL Cryptosporidium spp.
}

\author{
Heber Silva-Díaz ${ }^{1, a, d}$, Hamer Campos-Flores ${ }^{2, b}$, Jean Pier Llagas-Linares ${ }^{2, b}$, Dunalia LLatas-Cancino1,c
}

\begin{abstract}
RESUMEN
Con el objetivo de estimar la frecuencia de infección por coccidios intestinales en niños admitidos en un hospital de Perú, y comparar la tinción ácido-resistente modificada (TARM) y el ELISA para la detección de Cryptosporidium spp.; se realizó un estudio transversal entre octubre de 2014 y junio de 2015. Los coccidios se detectaron mediante la TARM y ELISA Cryptosporidium (kit r-Biopharm) en muestras seriadas de heces. De un total de 325 niños, el 5,5\% tuvieron algún coccidio intestinal, 3,7\% Cryptosporidium spp. (usando ambas técnicas) y 1,8\% Cyclospora cayetanensis (TARM). La TARM y ELISA mostraron una concordancia de 0,955 en la detección de Cryptosporidium spp. Se concluye que los coccidios intestinales son frecuentes en niños de la población estudiada; asimismo, ambas técnicas pueden usarse para la detección de Cryptosporidium spp., sin embargo, el menor costo y la capacidad de detectar otros coccidios ofrecen una ventaja a la TARM en la práctica diaria.
\end{abstract}

Palabras clave: coccidios intestinales, Cryptosporidium, Cyclospora, tinción ácido resistente, ensayo de inmunoadsorción enzimática (fuente: DeCS BIREME).

\section{INTESTINAL COCCIDIOSIS IN CHILDREN ADMITTED TO A HOSPITAL IN PERU AND COMPARISON OF TWO METHODS FOR DETECTING Cryptosporidium spp.}

\begin{abstract}
A cross-sectional study was done between October 2014 and June 2015 to estimate the frequency of infection due to intestinal coccidiosis in children admitted to a hospital in Peru, and compare the modified acid-fast staining procedure with the enzyme-linked immunoassay (ELISA) method for the detection of Cryptosporidium spp. Coccidia were detected using the modified acid-fast staining procedure and the Cryptosporidium ELISA method (kit r-Biopharm) on seriated stool samples. Out of a total of 325 children, $5.5 \%$ had some type of intestinal coccidiosis: $3.7 \%$ involved Cryptosporidium spp. (using both techniques) and $1.8 \%$ involved Cyclospora cayetanensis (using the modified acid-fast staining procedure). The modified acid-fast staining procedure and ELISA method revealed a 0.955 consistency in the detection of Cryptosporidium spp. In conclusion, intestinal coccidiosis is frequent in children based on the population studied; similarly, both techniques can be used to detect Cryptosporidium spp., but the modified acid-fast staining procedure has an advantage over the other because it is cost-effective and can detect other coccidia in everyday practice
\end{abstract}

Key words: intestinal coccidia, Cryptosporidium, Cyclospora, acid-fast stain, enzyme-linked immunosorbent assay (source: MeSH NLM)

\section{INTRODUCCIÓN}

Los coccidios intestinales (Cryptosporidium spp., Cyclospora cayetanesis y Cystoisospora belli) son parásitos protozoos apicomplejos de infección intracelular obligada, que afectan preferentemente la mucosa intestinal. La infección humana por coccidios es común en personas inmunocomprometidas e inmunocompetentes, siendo estos parásitos causa importante y emergente de diarrea alrededor del mundo, principalmente en niños ${ }^{(1-3)}$.

Numerosas características biológicas de los coccidios, tales como la sobrevivencia de los ooquistes durante largos

\footnotetext{
Laboratorio de Parasitología, Metaxénicas y Zoonosis, Hospital Regional Lambayeque. Lambayeque, Perú.

Facultad de Ciencias de la Salud, Universidad de Chiclayo. Lambayeque, Perú.

Biólogo; ${ }^{\mathrm{b}}$ tecnólogo médico; ${ }^{\mathrm{c}}$ maestro en Ciencias; ${ }^{\mathrm{d}}$ doctor en ciencias

Recibido: 29/08/2015 Aprobado: 24/08/2016
}

Citar como: Silva-Díaz H, Campos-Flores H, Llagas-Linares JP, LLatas-Cancino D. Coccidiosis intestinal en niños admitidos en un hospital de Perú y comparación de dos métodos para la detección del Cryptosporidium spp. Rev Peru Med Exp Salud Publica. 2016;33(4):739-44. doi:10.17843/rpmesp.2016.334.2560 
periodos de tiempo en el ambiente, la resistencia relativa a la desinfección con cloro ${ }^{(4)}$, y su transmisión zoonótica (algunas especies de Cryptosporidium spp.) ${ }^{(5,6)}$ y a través de agua y alimentos contaminados ${ }^{(7-9)}$; hacen de ellos potentes agentes infecciosos en países con escaso saneamiento básico como el Perú, país que ha sido considerado endémico, en particular para la cyclosporosis ${ }^{(10)}$. De hecho, los brotes epidémicos de coccidiosis intestinal de origen hídrico y alimentario son importantes en salud pública ${ }^{(3,10)}$. Las enfermedades intestinales de curso agudo y crónico de etiología desconocida son frecuentes en la práctica clínica ${ }^{(11)}$. De hecho, parte de los casos podrían deberse a infección por coccidios intestinales no diagnosticada, debido al escaso uso de pruebas específicas en los laboratorios, en especial en países en desarrollo como Perú. Al respecto, las pruebas coproparasitológicas rutinarias, como el examen microscópico directo presentan baja sensibilidad o son ineficaces para detectar los coccidios ${ }^{(12)}$. En este contexto, el diagnóstico oportuno y confiable de los coccidios intestinales cobra gran importancia para la prevención, vigilancia y tratamiento de la enfermedad.

Los ooquistes de los coccidios se detectan convencionalmente por microscopía óptica, en fresco ( $C$. cayetanensis y $C$. belli) o coloreados a través de varias técnicas que difieren en su sensibilidad y practicidad; sin embargo, la tinción ácido-resistente en frío (ziehlNeelsen modificado o Kinyoun) o caliente, ha prosperado hasta convertirse en la prueba de referencia (13-15) Asimismo, la microscopía de inmunofluorescencia son también usadas (14,16). Antígenos específicos en heces (coproantígenos) pueden ser detectados por métodos inmunológicos, como la inmunocromatografía y el enzimoinmuno análisis (ELISA) ${ }^{(17,18)}$, con ventajas de sensibilidad y especificidad respecto a la microscopía aún en debate. En los últimos años se han propuesto métodos moleculares altamente sensibles basados en la reacción en cadena de la polimerasa (PCR) para su detección, y en el caso de Cryptosporidium spp, diferenciación de especies ${ }^{(5,19)}$; sin embargo, debido al relativo alto costo aún no se han extendido para su uso en diagnóstico.

La frecuencia de la coccidiosis intestinal en población infantil es poco conocida en la región Lambayeque, lo que sumado a la baja sensibilidad o al uso de pruebas no específicas para su detección, no han permitido el diagnóstico y el oportuno tratamiento. En este sentido, es necesario promover métodos diagnósticos específicos, sencillos, sensibles, reproducibles y de bajo costo que puedan ser adoptados por los laboratorios.
El objetivo de este estudio fue describir la frecuencia y características clínicas asociadas a infección por coccidios intestinales en niños admitidos en el Hospital Regional Lambayeque, Perú; asimismo, comparar la tinción ácido resistente modificada (TARM) y ELISA coproantígenos en la detección de Cryptosporidium spp.

\section{EL ESTUDIO}

\section{DISEÑO Y POBLACIÓN DE ESTUDIO}

Estudio observacional de corte transversal realizado entre octubre de 2014 y junio de 2015 en niños admitidos en el Hospital Regional Lambayeque (HRL) en Chiclayo, Perú. El HRL es un hospital de alta complejidad, categoría III-1, que atiende pacientes referidos de la región Lambayeque y regiones aledañas.

Se incluyeron niños entre 6 meses y 10 años de edad atendidos en los servicios de consultorio externo, emergencia y medicina, a los cuales se les solicitó examen parasitológico seriado. Se excluyeron los niños que recibían tratamiento antimicrobiano al momento o días previos a la toma de muestra y aquellos con infección por el virus de la inmunodeficiencia humana. Se calculó un tamaño de muestra para estimar una proporción cuando la población es desconocida, se consideró un nivel de confianza de 0,95, un error de 0,035 y una proporción "P" esperada de 0,089 (12). Así mismo, el método de muestreo fue aleatorio simple. La recolección de las características epidemiológicas y clínicas (edad, sexo, procedencia, aspecto y consistencia de las heces, dolor abdominal, náusea-vómito y fiebre) fue a través de un cuestionario estructurado, aplicado por personal capacitado a los padres de familia o apoderados.

\section{DETECCIÓN DE LOS COCCIDIOS INTESTINALES}

Se recolectaron tres muestras seriadas de heces, se identificaron y fueron entregadas inmediatamente al Laboratorio de Parasitología, Metaxénicas y Zoonosis del HRL. En este lugar, cada muestra fue segregada y conservada con formalina al $10 \%$ una parte y congelada a $-70{ }^{\circ} \mathrm{C}$ la otra, para su posterior análisis mediante tinción ácido-resistente modificada (TARM) y ELISA para Cryptosporidium respectivamente. Los análisis fueron diarios para la TARM y semanal para el ELISA en forma ciega e independiente. Se consideró caso positivo cuando se observó ooquistes de coccidios al menos en una muestra mediante la TARM o ELISA coproantígenos. 
La detección de coccidios mediante TARM se realizó independientemente en tres muestras seriadas siguiendo el protocolo descrito por García et al. (1983) (13). La técnica consistió en extender finamente materia fecal previamente sedimentada por centrifugación. En muestras mucosas se trató previamente con $\mathrm{KOH}$ al $10 \%$ antes de la extensión. Después de secar y fijar, la extensión se coloreó con fucsina fenicada durante $5 \mathrm{~min}$, el que incluyó un calentamiento. Posteriormente, se decoloró por no más de $30 \mathrm{~s}$ y coloreó con azul de metileno por un minuto. Cada lectura microscópica fue realizada por dos microscopistas entrenados en el reconocimiento de los ooquistes de Cyclospora cayetanensis, Cryptosporidium spp. y Cystoisospora belli.

En cuanto al ensayo inmunológico por ELISA para la detección de Cryptosporidium spp., se usó el kit comercial Cryptosporidium r-Biopharm, USA, el que contenía anticuerpos para la detección cualitativa y específica de antígenos fecales del parásito. El kit mostró un certificado de calidad para cada lote usado y describió 96 y $100 \%$ de sensibilidad y especificidad, respectivamente, en referencia al PCR en tiempo real. Asimismo, excepto con Campylobacter coli, el kit no describe reacción cruzada con otros enteropatógenos. Previamente al ensayo se realizó un pool de las tres muestras de cada niño. El protocolo de ensayo de ELISA siguió las recomendaciones del fabricante y se consideró positivo a Cryptosporidium spp. índices superiores a 1,1; mientras que índices menores de 0,9 y 0,9 a 1,1 se interpretaron como negativo e indeterminado, respectivamente. En este último caso se repitió el examen solicitando al participante nueva serie de muestras.

\section{ANÁLISIS ESTADÍSTICO}

Se estimó la frecuencia de la coccidiosis intestinal y se realizó el análisis descriptivo de las variables independientes. Las pruebas de chi cuadrado, prueba exacta de Fisher y razón de probabilidades (odds ratio, OR) fueron calculadas para evaluar la asociación entre la coccidiosis y las variables independientes cualitativas. La comparación de las técnicas diagnósticas para Cryptosporidium (TARM y ELISA) se realizó mediante la diferencia de proporción de positivos detectados (rendimiento diagnóstico) por " $Z$ test" y del coeficiente de concordancia Kappa. Asimismo, se calculó la sensibilidad (S), especificidad (E), valor predictivo positivo (VPP) y negativo (VPN) del ELISA en referencia a la TARM, con el fin de evaluar su validez mediante la probabilidad de detección de los positivos y negativos.
Se consideró un nivel de confianza del $95 \%$ y un valor de $p<0,05$ como significativo. El análisis estadístico se realizó en los programas InfoStat/E versión 2008 y el GraphPad Prism versión 6.

\section{CONSIDERACIONES ÉTICAS}

El estudio fue revisado y aprobado por el Comité de Ética del Hospital Regional Lambayeque. La participación en el estudio fue voluntaria y aceptada a través de la firma de un consentimiento informado por parte del padre o apoderado y un asentimiento informado por los niños mayores de 8 años.

\section{RESULTADOS}

Se investigaron 975 muestras procedentes de 325 niños de entre 6 meses y 10 años de edad atendidos en el Hospital Regional Lambayeque, Chiclayo, Perú. La muestra tuvo una mediana de 4 años de edad y se caracterizó por el predominio del sexo masculino $(52,0 \%)$ y niños menores de 5 años (53,9\%). Las características clínicas que presentaron los niños se describen en la Tabla 1.

El análisis bivariado entre las variables independientes y la coccidiosis intestinal, mostró que presentar dolor abdominal y náusea-vómito estuvo asociado con la mayor frecuencia de la enfermedad; mientras que, la edad menor o igual de 5 años y la presencia de heces diarreicas no representaron asociación (Tabla 1).

El 5,5\% (18/325) de niños tuvo algún coccidio intestinal (IC 95\%: 3,0-8,0); 3,7\% (12/325) tuvo Cryptosporidiun spp. y $1,8 \%(6 / 325)$ C. cayetanensis. No se encontró ningún caso de infección por $C$. belli. En cuanto a la comparación entre las técnicas tinción ácido-resistente modificada (TARM) y ELISA en la detección de Cryptosporidium spp, los resultados se muestran en la Tabla 2. Ambas técnicas obtuvieron un rendimiento diagnóstico similar $(\mathrm{p}=0,999)$ y un índice de concordancia Kappa de 0,955 (IC 95\%: 0,846-1,000). Las estimaciones diagnósticas del ELISA en referencia a la TARM fueron: sensibilidad 100\% (IC $95 \%$ : 95,4100), especificidad 99,7\% (IC $95 \%$ : 98,9-100), valor predictivo positivo $91,7 \%$, valor predictivo negativo $100 \%$ y los coeficientes de probabilidad positivo y negativo fueron 314 y 0 respectivamente. 
Tabla 1. Características clínicas de los niños menores de 10 años atendidos en el Hospital Regional Lambayeque, Chiclayo 2015

\begin{tabular}{|c|c|c|c|c|}
\hline Variables & $\mathbf{N}(\%)$ & Coccidios/total (\%) & OR (IC 95\%) & Valor $p$ \\
\hline \multicolumn{5}{|l|}{ Sexo } \\
\hline Femenino & $223(48,0)$ & $8 / 156(5,1)$ & 1,00 & \\
\hline Masculino & $169(52,0)$ & $10 / 169(5,9)$ & $1,16(0,46-2,95)$ & $0,812^{*}$ \\
\hline \multicolumn{5}{|l|}{ Procedencia } \\
\hline Urbano & $223(68,6)$ & $12 / 223(5,4)$ & 1,00 & \\
\hline Rural & $102(31,4)$ & $6 / 102(5,9)$ & $0,58(0,21-1,60)$ & $0,332^{*}$ \\
\hline \multicolumn{5}{|l|}{ Grupo etario } \\
\hline 6 a 10 años & $150(46,1)$ & $10 / 175(6,7)$ & 1,00 & \\
\hline$\leq 5$ años & $175(53,9)$ & $8 / 175(4,6)$ & $0,67(0,26-1,70)$ & $0,471^{*}$ \\
\hline \multicolumn{5}{|l|}{ Color heces } \\
\hline Pardo & $194(59,7)$ & $11 / 194(5,7)$ & 1,00 & \\
\hline Verde & $56(17,2)$ & $3 / 56(5,4)$ & $0,94(0,25-3,50)$ & \\
\hline Marrón & $75(13,1)$ & $4 / 75(5,3)$ & $0,94(0,29-3,04)$ & $0,992^{* *}$ \\
\hline \multicolumn{5}{|l|}{ Aspecto heces } \\
\hline Semiformado & $197(60,6)$ & $7 / 197(3,6)$ & 1,00 & \\
\hline Formado & $35(10,8)$ & $2 / 35(5,7)$ & $1,65(0,33-8,27)$ & \\
\hline Diarreico & $93(28,6)$ & $9 / 93(9,7)$ & $2,91(1,05-8,07)$ & $0,103^{* *}$ \\
\hline \multicolumn{5}{|l|}{ Consistencia heces } \\
\hline Blando & $275(84,6)$ & $16 / 275(5,8)$ & 1,00 & \\
\hline Sólido & $23(7,1)$ & $0 / 23(0,0)$ & $0,34(0,02-5,76)$ & \\
\hline Líquido & $27(8,3)$ & $2 / 27(7,4)$ & $1,30(0,28-5,96)$ & $0,456^{* *}$ \\
\hline \multicolumn{5}{|l|}{ Síntomas } \\
\hline No & $161(49,5)$ & $5 / 161(3,1)$ & 1,00 & \\
\hline Sí & $164(51,5)$ & $13 / 164(7,9)$ & $2,69(0,93-7,72)$ & $0,087^{*}$ \\
\hline \multicolumn{5}{|l|}{ Dolor abdominal } \\
\hline No & $298(91,7)$ & $13 / 298(4,4)$ & 1,00 & \\
\hline Sí & $27(8,3)$ & $5 / 27(18,5)$ & $4,98(1,69-14,68)$ & $0,011^{*}$ \\
\hline \multicolumn{5}{|l|}{ Nausea-vómito } \\
\hline No & $309(95,1)$ & $14 / 309(4,5)$ & 1,00 & \\
\hline Sí & $16(4,9)$ & $4 / 16(25,0)$ & $7,02(2,12-23,31)$ & $0,008^{*}$ \\
\hline \multicolumn{5}{|l|}{ Fiebre } \\
\hline No & $302(92,9)$ & $16 / 302(5,3)$ & 1,00 & \\
\hline Sí & $23(7,1)$ & $2 / 23(8,7)$ & $1,70(0,42-6,91)$ & $0,627^{*}$ \\
\hline
\end{tabular}

* Prueba de Fisher exacta. ${ }^{* *}$ Prueba de chi cuadrado

\section{DISCUSIÓN}

La frecuencia de $5,5 \%$ de coccidiosis intestinal en niños, observada en nuestro estudio, fue inferior al reportado en dos estudios en niños de comunidades urbano marginales, uno en Lima, Perú $(8,9 \%)^{(12)}$ y otro en Caquetá, Colombia (19\%) ${ }^{(20)}$, las cuales fueron estimadas mediante coloraciones de Kinyoun y Kinyoun posesporulación, respectivamente. Sin embargo, si comparamos la frecuencia por especie de coccidio, los resultados obtenidos en nuestro estudio varían en mayor o menor grado al de otros estudios en Perú y América Latina. Estas diferencias podrían explicarse por las distintas condiciones sociodemográficas y ambientales que tuvieron las poblaciones estudiadas, así como por la naturaleza de la población (hospitalaria o comunitaria) del cual se tomó la muestra.

Así, el 3,7\% de Cryptosporidium spp. observado en este estudio, fue mayor al reportado en escolares de la 
Tabla 2. Frecuencia de detección de Cryptosporidium spp. por ELISA y tinción ácido-resistente modificada (TARM) en niños menores de 10 años atendidos en el Hospital Regional Lambayeque, Chiclayo 2015

\begin{tabular}{|c|c|c|c|c|c|c|c|}
\hline & \multicolumn{6}{|c|}{ TARM (Gold standard) } & \multirow{3}{*}{ Valor $\boldsymbol{p}^{*}$} \\
\hline & \multicolumn{2}{|c|}{ Positivo } & \multicolumn{2}{|c|}{ Negativo } & \multicolumn{2}{|c|}{ Total } & \\
\hline & $\mathbf{N}$ & $(\%)$ & $\mathbf{N}$ & $(\%)$ & $\mathbf{n}$ & $\%$ & \\
\hline \multicolumn{8}{|l|}{ ELISA } \\
\hline Positivo & 11 & $(3,4)$ & 1 & $(0,3)$ & 12 & $(3,7)$ & \multirow{3}{*}{0,999} \\
\hline Negativo & 0 & $(0,0)$ & 313 & $(96,3)$ & 313 & $(96,3)$ & \\
\hline Total & 11 & $(3,4)$ & 314 & $(96,6)$ & 325 & $(100,0)$ & \\
\hline
\end{tabular}

* Z test" para la diferencia de proporciones obtenidas por ambas técnicas

región Amazonas (1,9\%) ${ }^{(21)}$, pero menor al observado en Lima $(7,6 \%)^{(12)}$; mientras que en América Latina se han reportado frecuencias desde 1,9 a 46,8\% (20,22-24). La frecuencia de $C$. cayetanensis en un estudio en población general de Perú $(7,3 \text { y } 41,6 \%)^{(25)}$ y otro en niños de Colombia (4\%) ${ }^{(20)}$, fue mayor respecto al encontrado en este estudio (1,8\%). En cuanto a C. belli, no encontramos casos, sin embargo, en niños de Perú y Colombia se han reportado frecuencias de $1,3^{(12)}$ y $8 \%{ }^{(20)}$ respectivamente, lo que demuestra que $C$. belli también es un coccidio prevalente en población infantil inmunocompetente y asintomática. Cabe destacar que los estudios epidemiológicos basados en microscopía convencional tienden a subestimar la frecuencia real de infección debido a su menor sensibilidad.

El dolor abdominal y la náusea-vómito estuvo relacionado con la mayor frecuencia de infección por coccidios intestinales, no obstante, estas infecciones han sido asociadas a múltiples manifestaciones clínicas gastrointestinales y generales que varian según el coccidio y el hospedador ${ }^{(2,22,25)}$. La consistencia líquida de las heces (diarrea) no mostró asociación con la coccidiosis intestinal, hallazgo que también ha sido reportado en estudios previos en población inmunocompetente infectada con Cryptosporidium spp. (22-24). Asimismo, tampoco se encontró asociación con la fiebre, hecho inesperado, debido a que este signo se ha reportado como característica importante ${ }^{(22)}$.

En cuanto a la comparación de métodos para la detección de Cryptosporidium (TARM y ELISA), la alta concordancia y el mismo rendimiento diagnóstico encontrados bajo las condiciones de este estudio, evidencian que ambos métodos podrían utilizarse para detectar Cryptosporidium spp. en muestras fecales, tanto en estudios epidemiológicos como en el diagnóstico. En este contexto, la TARM es la técnica más usada en los laboratorios del mundo ${ }^{(14)}$. Sin embargo, en Perú aún no se ha adoptado, mientras que el ELISA es una potencial prueba alternativa o complementaria a la TARM debido a su disponibilidad comercial, pero que actualmente su uso no es extensivo. Estudios previos muestran al ELISA con una sensibilidad similar ${ }^{(16)}$ o menor ${ }^{(18)}$ respecto al TARM, lo que difiere de los resultados aquí presentados, lo que podría explicarse por la diferente intensidad de parasitación o cantidad de muestras examinadas.

La TARM tiene como ventaja su bajo costo, sencillez, adecuada sensibilidad, alta especificidad y detecta a los tres coccidios; sin embargo, requiere un microscopista experimentado y mucho tiempo en la observación microscópica para la identificación de los ooquistes, lo cual es una limitación cuando se procesan varias muestras en simultáneo ${ }^{(13,14)}$. Por otra parte, la técnica de ELISA para coproantígenos es adecuadamente sensible y específica, no requiere personal experimentado, es rápido, de fácil interpretación, y se puede examinar gran número de muestras a la vez ${ }^{(18)}$. No obstante, es más costoso que la TARM y no permite la detección de otros coccidios.

En conclusión, los coccidios intestinales son frecuentes en niños admitidos en el $\mathrm{HRL}$, lo que evidencia la importancia de esta parasitosis como problema de salud pública en la región y el país. Asimismo, debido a la alta concordancia, ambos métodos, TARM y ELISA, pueden usarse para la detección de Cryptosporidium spp. en estudios epidemiológicos y en el diagnóstico rutinario; sin embargo, el menor costo y la capacidad de detectar otros coccidios, ofrecen una ventaja a la TARM en la práctica diaria.

Contribuciones de autoría. HSD dio concepción y diseñó el estudio. HCF, JPLL y HSD participaron en la recolección de datos. DLC y HSD realizaron el análisis e interpretación de datos, redactaron y revisaron críticamente el artículo. Todos los autores revisaron las versiones preliminares del manuscrito y aprobaron la versión final del mismo.

Fuentes de financiamiento. el Hospital Regional Lambayeque, a través de las estrategias sanitarias Articulado Nutricional y Metaxénicas y Zoonosis, financió una parte, mientras que la otra fue autofinanciada. La Dirección de Investigación de la misma institución financió los gastos de uso de infraestructura y equipamiento de laboratorio.

Conflictos de interés. los autores declaran no tener conflictos de interés. 


\section{REFERENCIAS BIBLIOGRÁFICAS}

1. Archelli S, Kozubsky LE. Cyclospora cayetanensis: Un coccidio emergente. Acta bioquímica clínica Latinoam. 2012;46(4).

2. Legua P, Seas C. Cystoisospora and Cyclospora. Curr Opin Infect Dis. 2013;26(5):479-83.

3. Del Coco VF, Córdoba MA, Basualdo JA. Criptosporidiosis: una zoonosis emergente. Rev Argent Microbiol. 2009;41(3):185-96.

4. Chacín-Bonilla L, Barrios F. Cyclospora cayetanensis: biology, environmental distribution and transfer. Biomédica. 2011;31(1):132-44.

5. Neira OP, Muñoz SN, Wilson LG, Barthel MME, Rosales LMJ, Henríquez RC. Cryptosporidium species in immunodeficient and immunocompetent patients of Valparaíso: a descriptive study. Rev Chilena Infectol. 2012;29(1):63-71.

6. Helmy YA, Krücken J, Nöckler K, von Samson-Himmelstjerna G, Zessin KH. Molecular epidemiology of Cryptosporidium in livestock animals and humans in the Ismailia province of Egypt. Vet Parasitol. 2013;193(13):15-24.

7. Pérez-Cordón G, Rosales MJ, Valdez RA, Vargas-Vásquez F, Cordova O. Detección de parásitos intestinales en agua y alimentos de Trujillo, Perú. Rev Peru Med Exp Salud Publica. 2008;25(1):144-8.

8. Tananta VI, Chávez VA, Casas AE, Suárez AF, Serrano ME. Presencia de enteroparásitos en lechuga (Lactuca sativa) en establecimientos de consumo público de alimentos en el Cercado de Lima. Rev Inv Vet Perú. 2004;15(2):157-62.

9. Cárdenas M, Martínez R. Protozoarios parásitos de importancia en salud pública transportados por Musca domestica Linnaeus en Lima, Perú. Rev Peru Biol. 2004;11(2):149-52.

10. Chacín-Bonilla L. Epidemiology of Cyclospora cayetanensis: A review focusing in endemic areas. Acta Trop. 2010;115(3):181-93.

11. Beraun-Villa M, Valdez LM. Diarrea del viajero. Rev Medica Hered. 2013;24(1):54-61.

12. Huiza A, Espinoza Y, Rojas R, Sevilla C, Alva P, Verástegui $\mathrm{R}$, et al. Detección de coccidios en niños asintomáticos mediante esporulación de muestras fecales. An la Fac Med. 2004;65(4):239-42.

13. Garcia LS, Bruckner DA, Brewer TC, Shimizu RY. Techniques for the recovery and identification of Cryptosporidium oocysts from stool specimens. J Clin Microbiol. 1983;18(1):185-90.

14. Manser M, Granlund M, Edwards H, Saez A, Petersen E, Evengard B, et al. Detection of Cryptosporidium and Giardia in clinical laboratories in Europe--a comparative study. Clin Microbiol Infect. 2014;20(1):O65-71.

15. Galvan-Díaz AL, Herrera-Jaramillo V, Santos-Rodriguez ZM, DelgadoNaranjo M. Modified Ziehl-Neelsen and modified Safranin staining for diagnosing Cyclospora cayetanensis. Rev Salud Publica (Bogota). 2008;10(3):488-93.

16. Kehl KS, Cicirello H, Havens PL. Comparison of four different methods for detection of Cryptosporidium species. J Clin Microbiol. 1995;33(2):416-8.

17. Youn S, Kabir M, Haque R, Petri WA. Evaluation of a screening test for detection of Giardia and Cryptosporidium parasites. J Clin Microbiol. 2009;47(2):451-2.

18. Johnston SP, Ballard MM, Beach MJ, Causer L, Wilkins PP. Evaluation of three commercial assays for detection of Giardia and Cryptosporidium organisms in fecal specimens. J Clin Microbiol. 2003;41(2):623-6.

19. Checkley W, White AC, Jaganath D, Arrowood MJ, Chalmers RM, Chen XM, et al. A review of the global burden, novel diagnostics, therapeutics, and vaccine targets for Cryptosporidium. Lancet Infect Dis. 2015;15(1):85-94.

20. Lucero-Garzón TA, Álvarez-Motta LA, Chicue-López JF, López-Zapata D, Mendoza-Bergaño CA. Parasitosis intestinal y factores de riesgo en niños de los asentamientos subnormales, FlorenciaCaquetá, Colombia. Rev Fac Nac Salud Pública. 2015;33(2):171-80.

21. Ibáñez N, Jara C, Guerra A, Díaz E. Prevalencia del enteroparasitismo en escolares de comunidades nativas del Alto Marañon, Amazonas, Perú. Rev Peru Med Exp Salud Publica. 2004;21(3):126-33.

22. De la Ossa Merlano N, Falconar A, Llinás Solano HJ, Romero Vivas CM. Clinical manifestations and risk factors associated with Cryptosporidium spp. infections in patients from Barranquilla and three "municipios" of Atlántico (Colombia). Rev Salud Uninorte. 2007;23(1):19-31.

23. Bayona RM, Avendaño VC, Amaya MÁ. Epidemiologig characterization of cryptosporidiosis in infant population at the Sabana Centro (Cundinamarca). Rev UDCA Actual \& amp; Divulg Científica; 2011;14(1):713.

24. Arango M, Rodríguez DA, Prada NE. Frecuency of Cryptosporidium spp. in childres's stool of one month to thirteen years old in a local Colombian hospital. Colomb Med. 2006;37(2):121-5.

25. Burstein Alva S. Ciclosporosis: una parasitosis emergente (I). Aspectos clínicos y epidemiológicos. Rev Gastroenterol del Perú. 2005;25(4):328-35.

Correspondencia: Heber Silva-Díaz

Dirección: Av. Vía de Evitamiento Norte con Av. El Progreso. Dirección de Investigación del Hospital Regional Lambayeque. Chiclayo, Perú.

Correo electrónico: h.silvadiaz@hotmail.com 